\title{
Common fixed points for a pair of mappings satisfying contractive conditions of integral type
}

\author{
Zeqing Liu', Xiaochen Zou', Shin Min Kang ${ }^{2 *}$ and Jeong Sheok Ume ${ }^{3}$ \\ Dedicated to Professor Shih-sen Chang on his 80th birthday.
}

\author{
${ }^{*}$ Correspondence: \\ smkang@gnu.ac.kr \\ ${ }^{2}$ Department of Mathematics and \\ the RINS, Gyeongsang National \\ University, Jinju, 660-701, Korea \\ Full list of author information is \\ available at the end of the article
}

\begin{abstract}
Four common fixed point theorems for a pair of weakly compatible mappings satisfying contractive conditions of integral type in metric spaces are proved. The existence result of bounded solutions for a system of functional equations arising in dynamic programming is discussed by using one of the common fixed point theorems obtained in this paper. An example is given to illustrate that our results extend properly two fixed point theorems due to Branciari and Rhoades.

MSC: $54 \mathrm{H} 25$
\end{abstract}

Keywords: contractive mappings of integral type; weakly compatible mappings; common fixed point; system of functional equations; dynamic programming

\section{Introduction and preliminaries}

In 2002, Branciari [1] proved an interesting fixed point theorem for a single-valued contractive mapping of integral type satisfying an analog of the Banach contraction principle in metric spaces. Afterwards many researchers [2-20] extended the result of Branciari and obtained a lot of fixed point and common fixed point theorems for various single-valued and multi-valued mappings involving a large amount of general contractive conditions of integral type in metric spaces, modular spaces, symmetric spaces, fuzzy metric spaces, cone metric spaces, uniform spaces, and Hausdorff topological spaces etc.

Liu et al. [12] and Rhoades [15] got the existence, uniqueness, and iterative approximations of fixed points for general contractive mappings of integral type, Djoudi and Merghadi [8] and Vijayaraju et al. [20] showed several common fixed point theorems for a pair of weakly compatible mappings satisfying certain contractive mappings of integral type, Altun and Türkoğlu [3], Altun et al. [5] and Djoudi and Aliouche [7] discussed a few common fixed point theorems for two pairs of weakly compatible mappings satisfying an implicit relation and contractive conditions of integral type, respectively, Suzuki [19] proved that Meir-Keeler contractions of integral type are still Meir-Keeler contractions, Jachymski [10] discussed that most contractive conditions of integral type given recently by many authors coincide with classical ones and got a new contractive condition of integral type which is independent of classical ones, and Sintunavarat and Kumam [17, 18] 
proved Gregus-type common fixed point theorems for tangential multi-valued mappings satisfying strict general contractive conditions of integral type in metric spaces.

Beygmohammadi and Razani [6], Hussain and Salimi [9], and Mongkolkeha and Kumam [13] presented more general fixed point and common fixed point theorems for some integral-type contractions in modular spaces. Murthy et al. [14] proved common fixed point theorems for different variant of compatible mappings, satisfying a contractive condition of integral type in fuzzy metric spaces. De la Sen [16] investigated the existence of fixed points and best proximity points of $p$-cyclic self-mappings in a set of subsets of a certain uniform space under integral-type contractive conditions. Khojasteh et al. [11] obtained a fixed point theorem of an integral-type contraction in complete cone metric spaces. Aliouche [2] proved a common fixed point theorem for two pairs of weakly compatible mappings satisfying a contractive condition of integral type in symmetric spaces. Altun and Türkoğlu [4] established two fixed point and common fixed point theorems for mappings satisfying contractive conditions of integral type in Hausdorff $d$-complete topological spaces.

However, to the best of our knowledge, no one studied the existence and uniqueness problems of common fixed points for a pair of contractive mappings of integral type satisfying (2.1), (2.13), (2.20), and (2.22), respectively.

The aim of this paper is to show the existence and uniqueness of common fixed points for the four kinds of weakly compatible mappings (2.1), (2.13), (2.20), and (2.22) in metric spaces under weaker conditions. As an application, we use Theorem 2.1 to study solvability of system of functional equations (2.26). Our results extend, improve, and unify Theorem 2.1 of Branciari [1], Theorems 5.1-5.3 of Liu et al. [12], Theorem 2 of Rhoades [15], Theorem 1 of Bhakta and Mitra [21], Theorem 3.1 of Liu and Kang [22], and Theorem 3.2 of Liu et al. [23]. A nontrivial example with uncountably many points is included.

Throughout this paper we assume that $\mathbb{R}^{+}=[0,+\infty), \mathbb{N}_{0}=\{0\} \cup \mathbb{N}$, where $\mathbb{N}$ denotes the set of all positive integers, and

$$
\begin{aligned}
& \Phi=\left\{\varphi: \varphi: \mathbb{R}^{+} \rightarrow \mathbb{R}^{+} \text {satisfies the requirement that } \varphi\right. \text { is Lebesgue integrable, } \\
& \left.\quad \text { summable on each compact subset of } \mathbb{R}^{+} \text {and } \int_{0}^{\varepsilon} \varphi(t) d t>0 \text { for each } \varepsilon>0\right\}, \\
& \Psi=\left\{\psi: \psi: \mathbb{R}^{+} \rightarrow \mathbb{R}^{+}\right. \text {is upper semi-continuous, } \\
& \\
& \psi(0)=0 \text { and } \psi(t)>0 \text { for each } t>0\} .
\end{aligned}
$$

Recall that a pair of self-mappings $f$ and $g$ in a metric space $(X, d)$ are said to be weakly compatible if they commute at their coincidence points.

The following lemma plays an important role in this paper.

Lemma 1.1 ([12]) Let $\varphi \in \Phi$ and $\left\{r_{n}\right\}_{n \in \mathbb{N}}$ be a nonnegative sequence with $\lim _{n \rightarrow \infty} r_{n}=a$. Then

$$
\lim _{n \rightarrow \infty} \int_{0}^{r_{n}} \varphi(t) d t=\int_{0}^{a} \varphi(t) d t
$$


Lemma 1.2 ([22]) Let E be a set, $p$ and $q: E \rightarrow \mathbb{R}$ be mappings. If opt $_{y \in E} p(y)$ and opt $_{y \in E} q(y)$ are bounded, then

$$
\left|\operatorname{opt}_{y \in E} p(y)-\underset{y \in E}{\operatorname{opt}} q(y)\right| \leq \sup _{y \in E}|p(y)-q(y)| .
$$

\section{Common fixed point theorems}

Now we show the existence and uniqueness of common fixed points for four classes of weakly compatible mappings satisfying contractive conditions of integral type.

Theorem 2.1 Let $(X, d)$ be a metric space and let $f$ and $g$ be weakly compatible selfmappings on $X$ satisfying

$$
\int_{0}^{d(f x, f y)} \varphi(t) d t \leq \psi\left(\int_{0}^{M_{1}(x, y)} \varphi(t) d t\right), \quad \forall x, y \in X
$$

where $(\varphi, \psi) \in \Phi \times \Psi$ and

$$
\begin{aligned}
M_{1}(x, y)= & \max \left\{d(g x, g y), d(f x, g x), d(f y, g y), \frac{d(f x, g y)+d(f y, g x)}{2},\right. \\
& \left.\frac{d(f x, g y) d(f y, g x)}{1+d(g x, g y)}, \frac{d(f x, g x) d(f y, g x)}{2[1+d(g x, g y)]}, \frac{d(f y, g y) d(f x, g y)}{2[1+d(g x, g y)]}\right\}, \quad \forall x, y \in X .
\end{aligned}
$$

If $f(X) \subseteq g(X)$ and $g(X)$ is complete, then $f$ and $g$ have a unique common fixed point in $X$.

Proof Firstly we prove that $f$ and $g$ have at most one common fixed point in $X$. Suppose that $f$ and $g$ possess two common fixed points $a, b \in X$ and $a \neq b$. It follows from (2.1), (2.2), and $(\varphi, \psi) \in \Phi \times \Psi$ that

$$
\begin{aligned}
M_{1}(a, b)= & \max \left\{d(g a, g b), d(f a, g a), d(f b, g b), \frac{d(f a, g b)+d(f b, g a)}{2},\right. \\
& \left.\frac{d(f a, g b) d(f b, g a)}{1+d(g a, g b)}, \frac{d(f a, g a) d(f b, g a)}{2[1+d(g a, g b)]}, \frac{d(f b, g b) d(f a, g b)}{2[1+d(g a, g b)]}\right\} \\
= & \max \left\{d(a, b), 0,0, d(a, b), \frac{d^{2}(a, b)}{1+d(a, b)}, 0,0\right\} \\
= & d(a, b)
\end{aligned}
$$

and

$$
\begin{aligned}
\int_{0}^{d(a, b)} \varphi(t) d t & =\int_{0}^{d(f a, f b)} \varphi(t) d t \leq \psi\left(\int_{0}^{M_{1}(a, b)} \varphi(t) d t\right) \\
& =\psi\left(\int_{0}^{d(a, b)} \varphi(t) d t\right) \\
& <\int_{0}^{d(a, b)} \varphi(t) d t,
\end{aligned}
$$

which is impossible.

Secondly we show that $f$ and $g$ have a common fixed point in $X$. Let $x_{0}$ be an arbitrary point in $X$. Since $f(X) \subseteq g(X)$, it follows that there exists a sequence $\left\{x_{n}\right\}_{n \in \mathbb{N}_{0}}$ in $X$ satisfying $f x_{n}=g x_{n+1}$ for each $n \in \mathbb{N}_{0}$. Put $d_{n}=d\left(f x_{n}, f x_{n+1}\right)$ for all $n \in \mathbb{N}_{0}$. 
Assume that $d_{n_{0}}=0$ for some $n \in \mathbb{N}_{0}$. It follows that

$$
f x_{n_{0}}=f x_{n_{0}+1}=g x_{n_{0}+1}
$$

Because $f$ and $g$ are weakly compatible, by (2.3) we get

$$
f^{2} x_{n_{0}+1}=f g x_{n_{0}+1}=g f x_{n_{0}+1}=g^{2} x_{n_{0}+1} .
$$

Now we assert that $f x_{n_{0}+1}=f^{2} x_{n_{0}+1}$. Otherwise we infer that in view of (2.1)-(2.4) and $(\varphi, \psi) \in \Phi \times \Psi$

$$
\begin{aligned}
& M_{1}\left(f x_{n_{0}+1}, x_{n_{0}+1}\right) \\
&=\max \left\{d\left(g f x_{n_{0}+1}, g x_{n_{0}+1}\right), d\left(f^{2} x_{n_{0}+1}, g f x_{n_{0}+1}\right), d\left(f x_{n_{0}+1}, g x_{n_{0}+1}\right),\right. \\
& \frac{d\left(f^{2} x_{n_{0}+1}, g x_{n_{0}+1}\right)+d\left(f x_{n_{0}+1}, g f x_{n_{0}+1}\right)}{2}, \frac{d\left(f^{2} x_{n_{0}+1}, g x_{n_{0}+1}\right) d\left(f x_{n_{0}+1}, g f x_{n_{0}+1}\right)}{1+d\left(g f x_{n_{0}+1}, g x_{n_{0}+1}\right)}, \\
&\left.\frac{d\left(f^{2} x_{n_{0}+1}, g f x_{n_{0}+1}\right) d\left(f x_{n_{0}+1}, g f x_{n_{0}+1}\right)}{2\left[1+d\left(g f x_{n_{0}+1}, g x_{n_{0}+1}\right)\right]}, \frac{d\left(f x_{n_{0}+1}, g x_{n_{0}+1}\right) d\left(f^{2} x_{n_{0}+1}, g x_{n_{0}+1}\right)}{2\left[1+d\left(g f x_{n_{0}+1}, g x_{n_{0}+1}\right)\right]}\right\} \\
&= \max \left\{d\left(f^{2} x_{n_{0}+1}, f x_{n_{0}+1}\right), 0,0, d\left(f^{2} x_{n_{0}+1}, f x_{n_{0}+1}\right),\right. \\
&\left.\frac{d\left(f^{2} x_{n_{0}+1}, f x_{n_{0}+1}\right) d\left(f x_{n_{0}+1}, f^{2} x_{n_{0}+1}\right)}{1+d\left(f^{2} x_{n_{0}+1}, f x_{n_{0}+1}\right)}, 0,0\right\} \\
&= d\left(f^{2} x_{n_{0}+1}, f x_{n_{0}+1}\right)
\end{aligned}
$$

and

$$
\begin{aligned}
\int_{0}^{d\left(f^{2} x_{n_{0}+1} f x_{n_{0}+1}\right)} \varphi(t) d t & \leq \psi\left(\int_{0}^{M_{1}\left(f x_{n_{0}+1}, x_{n_{0}+1}\right)} \varphi(t) d t\right) \\
& =\psi\left(\int_{0}^{d\left(f^{2} x_{n_{0}+1} f x_{n_{0}+1}\right)} \varphi(t) d t\right) \\
& <\int_{0}^{d\left(f^{2} x_{n_{0}+1} f x_{n_{0}+1}\right)} \varphi(t) d t
\end{aligned}
$$

which is absurd. Therefore $f x_{n_{0}+1}=f^{2} x_{n_{0}+1}$, which together with (2.3) means that $f x_{n_{0}+1}$ is a common fixed point of $f$ and $g$ in $X$.

Assume that $d_{n} \neq 0$ for all $n \in \mathbb{N}_{0}$. Observe that

$$
\frac{d_{n-1} d\left(f x_{n+1}, f x_{n-1}\right)}{2\left(1+d_{n-1}\right)} \leq \frac{d\left(f x_{n+1}, f x_{n-1}\right)}{2} \leq \frac{d_{n-1}+d_{n}}{2} \leq \max \left\{d_{n-1}, d_{n}\right\}, \quad \forall n \in \mathbb{N} .
$$

It follows from (2.2) and (2.5) that

$$
\begin{aligned}
& M_{1}\left(x_{n}, x_{n+1}\right) \\
& \quad=\max \left\{d\left(g x_{n}, g x_{n+1}\right), d\left(f x_{n}, g x_{n}\right), d\left(f x_{n+1}, g x_{n+1}\right),\right.
\end{aligned}
$$




$$
\begin{aligned}
& \frac{d\left(f x_{n}, g x_{n+1}\right)+d\left(f x_{n+1}, g x_{n}\right)}{2}, \frac{d\left(f x_{n}, g x_{n+1}\right) d\left(f x_{n+1}, g x_{n}\right)}{1+d\left(g x_{n}, g x_{n+1}\right)}, \\
& \left.\frac{d\left(f x_{n}, g x_{n}\right) d\left(f x_{n+1}, g x_{n}\right)}{2\left[1+d\left(g x_{n}, g x_{n+1}\right)\right]}, \frac{d\left(f x_{n+1}, g x_{n+1}\right) d\left(f x_{n}, g x_{n+1}\right)}{2\left[1+d\left(g x_{n}, g x_{n+1}\right)\right]}\right\} \\
= & \max \left\{d\left(f x_{n-1}, f x_{n}\right), d\left(f x_{n}, f x_{n-1}\right), d\left(f x_{n+1}, f x_{n}\right),\right. \\
& \frac{d\left(f x_{n}, f x_{n}\right)+d\left(f x_{n+1}, f x_{n-1}\right)}{2}, \frac{d\left(f x_{n}, f x_{n}\right) d\left(f x_{n+1}, f x_{n-1}\right)}{1+d\left(f x_{n-1}, f x_{n}\right)}, \\
& \left.\frac{d\left(f x_{n}, f x_{n-1}\right) d\left(f x_{n+1}, f x_{n-1}\right)}{2\left[1+d\left(f x_{n-1}, f x_{n}\right)\right]}, \frac{d\left(f x_{n+1}, f x_{n}\right) d\left(f x_{n}, f x_{n}\right)}{2\left[1+d\left(f x_{n-1}, f x_{n}\right)\right]}\right\} \\
= & \max \left\{d_{n-1}, d_{n-1}, d_{n}, \frac{d\left(f x_{n+1}, f x_{n-1}\right)}{2}, 0, \frac{d_{n-1} d\left(f x_{n+1}, f x_{n-1}\right)}{2\left(1+d_{n-1}\right)}, 0\right\} \\
= & \max \left\{d_{n-1}, d_{n}\right\}, \quad \forall n \in \mathbb{N} .
\end{aligned}
$$

If $d_{n}>d_{n-1}$ for some $n \in \mathbb{N}$, using (2.1), (2.6), and $(\varphi, \psi) \in \Phi \times \Psi$, we conclude that

$$
\begin{aligned}
\int_{0}^{d_{n}} \varphi(t) d t & =\int_{0}^{d \int\left(x_{n}, f x_{n+1}\right)} \varphi(t) d t \leq \psi\left(\int_{0}^{M_{1}\left(x_{n}, x_{n+1}\right)} \varphi(t) d t\right) \\
& =\psi\left(\int_{0}^{d_{n}} \varphi(t) d t\right) \\
& <\int_{0}^{d_{n}} \varphi(t) d t
\end{aligned}
$$

which is a contradiction. Hence $d_{n} \leq d_{n-1}$ for each $n \in \mathbb{N}$. Consequently, the sequence $\left\{d_{n}\right\}_{n \in \mathbb{N}_{0}}$ is nondecreasing and bounded, which imply that there exists a constant $W$ with $\lim _{n \rightarrow \infty} d_{n}=W \geq 0$.

Next we show that $W=0$. Otherwise $W>0$. Taking the upper limit in (2.1) and using (2.6), $(\varphi, \psi) \in \Phi \times \Psi$, and Lemma 1.1, we infer that

$$
\begin{aligned}
\int_{0}^{W} \varphi(t) d t & =\limsup _{n \rightarrow \infty} \int_{0}^{d_{n}} \varphi(t) d t=\limsup _{n \rightarrow \infty} \int_{0}^{d\left(f x_{n}, f x_{n+1}\right)} \varphi(t) d t \\
& \leq \limsup _{n \rightarrow \infty} \psi\left(\int_{0}^{M_{1}\left(x_{n}, x_{n+1}\right)} \varphi(t) d t\right)=\limsup _{n \rightarrow \infty} \psi\left(\int_{0}^{d_{n-1}} \varphi(t) d t\right) \\
& \leq \psi\left(\limsup _{n \rightarrow \infty} \int_{0}^{d_{n-1}} \varphi(t) d t\right)=\psi\left(\int_{0}^{W} \varphi(t) d t\right) \\
& <\int_{0}^{W} \varphi(t) d t,
\end{aligned}
$$

which is impossible. Therefore, $W=0$, that is,

$$
\lim _{n \rightarrow \infty} d_{n}=0
$$

Now we prove that $\left\{f x_{n}\right\}_{n \in \mathbb{N}_{0}}$ is a Cauchy sequence. Suppose that $\left\{f x_{n}\right\}_{n \in \mathbb{N}_{0}}$ is not a Cauchy sequence, which means that there exist a constant $\varepsilon>0$ and two sequences 
$\{m(p)\}_{n \in \mathbb{N}}$ and $\{n(p)\}_{n \in \mathbb{N}}$ in $N$ such that $m(p)<n(p)<m(p+1)$ and

$$
d\left(f x_{m(p)}, f x_{n(p)}\right) \geq \varepsilon, \quad d\left(f x_{m(p)}, f x_{n(p)-1}\right)<\varepsilon, \quad \forall p \in \mathbb{N} .
$$

Note that

$$
\begin{aligned}
& d\left(f x_{m(p)}, f x_{n(p)}\right) \leq d\left(f x_{n(p)-1}, f x_{m(p)}\right)+d_{n(p)-1}, \quad \forall p \in \mathbb{N} ; \\
& \left|d\left(f x_{m(p)}, f x_{n(p)}\right)-d\left(f x_{m(p)}, f x_{n(p)-1}\right)\right| \leq d_{n(p)-1}, \quad \forall p \in \mathbb{N} ; \\
& \left|d\left(f x_{m(p)}, f x_{n(p)}\right)-d\left(f x_{m(p)-1}, f x_{n(p)}\right)\right| \leq d_{m(p)-1}, \quad \forall p \in \mathbb{N} ; \\
& \left|d\left(f x_{m(p)-1}, f x_{n(p)-1}\right)-d\left(f x_{m(p)-1}, f x_{n(p)}\right)\right| \leq d_{n(p)-1}, \quad \forall p \in \mathbb{N} .
\end{aligned}
$$

By virtue of (2.7)-(2.9), we deduce that

$$
\begin{aligned}
\varepsilon & =\lim _{p \rightarrow \infty} d\left(f x_{n(p)}, f x_{m(p)}\right)=\lim _{p \rightarrow \infty} d\left(f x_{m(p)}, f x_{n(p)-1}\right) \\
& =\lim _{p \rightarrow \infty} d\left(f x_{m(p)-1}, f x_{n(p)}\right)=\lim _{p \rightarrow \infty} d\left(f x_{m(p)-1}, f x_{n(p)-1}\right) .
\end{aligned}
$$

In light of (2.1), (2.2), (2.7), (2.10), $(\varphi, \psi) \in \Phi \times \Psi$, and Lemma 1.1, we conclude that

$$
\begin{aligned}
\lim _{p \rightarrow \infty} & M_{1}\left(x_{m(p)}, x_{n(p)}\right) \\
= & \lim _{p \rightarrow \infty} \max \left\{d\left(g x_{m(p)}, g x_{n(p)}\right), d\left(f x_{m(p)}, g x_{m(p)}\right), d\left(f x_{n(p)}, g x_{n(p)}\right),\right. \\
& \frac{d\left(f x_{m(p)}, g x_{n(p)}\right)+d\left(f x_{n(p)}, g x_{m(p)}\right)}{2}, \frac{d\left(f x_{m(p)}, g x_{n(p)}\right) d\left(f x_{n(p)}, g x_{m(p)}\right)}{1+d\left(g x_{m(p)}, g x_{n(p)}\right)}, \\
& \left.\frac{d\left(f x_{m(p)}, g x_{m(p)}\right) d\left(f x_{n(p)}, g x_{m(p)}\right)}{2\left[1+d\left(g x_{m(p)}, g x_{n(p)}\right)\right]}, \frac{d\left(f x_{n(p)}, g x_{n(p)}\right) d\left(f x_{m(p)}, g x_{n(p)}\right)}{2\left[1+d\left(g x_{m(p)}, g x_{n(p)}\right)\right]}\right\} \\
= & \lim _{p \rightarrow \infty} \max \left\{d\left(f x_{m(p)-1}, f x_{n(p)-1}\right), d\left(f x_{m(p)}, f x_{m(p)-1}\right), d\left(f x_{n(p)}, f x_{n(p)-1}\right),\right. \\
& \frac{d\left(f x_{m(p)}, f x_{n(p)-1}\right)+d\left(f x_{n(p)}, f x_{m(p)-1}\right)}{2}, \frac{d\left(f x_{m(p)}, f x_{n(p)-1}\right) d\left(f x_{n(p)}, f x_{m(p)-1}\right)}{1+d\left(f x_{m(p)-1}, f x_{n(p)-1}\right)}, \\
& \left.\frac{d\left(f x_{m(p)}, f x_{m(p)-1}\right) d\left(f x_{n(p)}, f x_{m(p)-1}\right)}{2\left[1+d\left(f x_{m(p)-1}, f x_{n(p)-1}\right)\right]}, \frac{d\left(f x_{n(p)}, f x_{n(p)-1}\right) d\left(f x_{m(p)}, f x_{n(p)-1}\right)}{2\left[1+d\left(f x_{m(p)-1}, f x_{n(p)-1}\right)\right]}\right\} \\
= & \max \left\{\varepsilon, 0,0, \varepsilon, \frac{\varepsilon^{2}}{1+\varepsilon}, 0,0\right\} \\
= & \varepsilon
\end{aligned}
$$

and

$$
\begin{aligned}
\int_{0}^{\varepsilon} \varphi(t) d t & =\limsup _{p \rightarrow \infty} \int_{0}^{d\left(f x_{m(p)} f x_{n(p)}\right)} \varphi(t) d t \leq \limsup _{p \rightarrow \infty} \psi\left(\int_{0}^{M_{1}\left(x_{m(p)}, x_{n(p)}\right)} \varphi(t) d t\right) \\
& \leq \psi\left(\limsup _{p \rightarrow \infty} \int_{0}^{M_{1}\left(x_{\left.m(p), x_{n(p)}\right)}\right.} \varphi(t) d t\right)=\psi\left(\int_{0}^{\varepsilon} \varphi(t) d t\right) \\
& <\int_{0}^{\varepsilon} \varphi(t) d t,
\end{aligned}
$$


which is a contradiction. Hence $\left\{x_{n}\right\}_{n \in \mathbb{N}_{0}}$ is a Cauchy sequence. Since $g(X)$ is complete, there exist $a, z \in X$ with

$$
\lim _{n \rightarrow \infty} f x_{n}=\lim _{n \rightarrow \infty} g x_{n}=a=g z
$$

Suppose that $f z \neq a$. Making use of (2.1), (2.2), (2.11), and $(\varphi, \psi) \in \Phi \times \Psi$ and Lemma 1.1, we arrive at

$$
\begin{aligned}
\lim _{n \rightarrow \infty} M_{1}\left(z, x_{n}\right)= & \lim _{n \rightarrow \infty} \max \left\{d\left(g z, g x_{n}\right), d(f z, g z), d\left(f x_{n}, g x_{n}\right),\right. \\
& \frac{d\left(f z, g x_{n}\right)+d\left(f x_{n}, g z\right)}{2}, \frac{d\left(f z, g x_{n}\right) d\left(f x_{n}, g z\right)}{1+d\left(g z, g x_{n}\right)}, \\
& \left.\frac{d(f z, g z) d\left(f x_{n}, g z\right)}{2\left[1+d\left(g z, g x_{n}\right)\right]}, \frac{d\left(f x_{n}, g x_{n}\right) d\left(f z, g x_{n}\right)}{2\left[1+d\left(g z, g x_{n}\right)\right]}\right\} \\
= & \max \left\{d(a, a), d(f z, a), d(a, a), \frac{d(f z, a)+d(a, a)}{2},\right. \\
& \left.\frac{d(f z, a) d(a, a)}{1+d(a, a)}, \frac{d(f z, a) d(a, a)}{2[1+d(a, a)]}, \frac{d(a, a) d(f z, a)}{2[1+d(a, a)]}\right\} \\
= & \max \left\{0, d(f z, a), 0, \frac{d(f z, a)}{2}, 0,0,0\right\} \\
= & d(f z, a)
\end{aligned}
$$

and

$$
\begin{aligned}
\int_{0}^{d(f z, a)} \varphi(t) d t & =\limsup _{n \rightarrow \infty} \int_{0}^{d\left(f z, f x_{n}\right)} \varphi(t) d t \leq \limsup _{n \rightarrow \infty} \psi\left(\int_{0}^{M_{1}\left(z, x_{n}\right)} \varphi(t) d t\right) \\
& \leq \psi\left(\limsup _{n \rightarrow \infty} \int_{0}^{M_{1}\left(z, x_{n}\right)} \varphi(t) d t\right)=\psi\left(\int_{0}^{d(f z, a)} \varphi(t) d t\right) \\
& <\int_{0}^{d(f z, a)} \varphi(t) d t,
\end{aligned}
$$

which is a contradiction. Thus $a=f z=g z$. Since $f$ and $g$ are weakly compatible, it follows that

$$
f a=f^{2} z=f g z=g f z=g^{2} z=g a .
$$

Suppose that $a \neq f a$. In view of (2.1), (2.2) (2.12), and $(\varphi, \psi) \in \Phi \times \Psi$, we infer that

$$
\begin{aligned}
M_{1}(z, g z)= & \max \left\{d(g z, g g z), d(f z, g z), d(f g z, g g z), \frac{d(f z, g g z)+d(f g z, g z)}{2},\right. \\
& \left.\frac{d(f z, g g z) d(f g z, g z)}{1+d(g z, g g z)}, \frac{d(f z, g z) d(f g z, g z)}{2[1+d(g z, g g z)]}, \frac{d(f g z, g g z) d(f z, g g z)}{2[1+d(g z, g g z)]}\right\} \\
= & \max \left\{d(a, f a), 0,0, \frac{d(a, f a)+d(f a, a)}{2}, \frac{d^{2}(a, f a)}{1+d(a, f a)}, 0,0\right\} \\
= & d(a, f a)
\end{aligned}
$$


and

$$
\begin{aligned}
\int_{0}^{d(a, f a)} \varphi(t) d t & =\int_{0}^{d(f z, f g z)} \varphi(t) d t \leq \psi\left(\int_{0}^{M_{1}(z, g z)} \varphi(t) d t\right) \\
& =\psi\left(\int_{0}^{d(a, f a)} \varphi(t) d t\right) \\
& <\int_{0}^{d(a, f a)} \varphi(t) d t,
\end{aligned}
$$

which is absurd. Hence $a=f a$. It follows from (2.12) that $f$ and $g$ have a common fixed point $a \in X$. This completes the proof.

Theorem 2.2 Let $(X, d)$ be a metric space and let $f$ and $g$ be weakly compatible selfmappings on $X$ satisfying

$$
\int_{0}^{d(f x, f y)} \varphi(t) d t \leq \psi\left(\int_{0}^{M_{2}(x, y)} \varphi(t) d t\right), \quad \forall x, y \in X
$$

where $(\varphi, \psi) \in \Phi \times \Psi$ and

$$
\begin{aligned}
M_{2}(x, y)= & \max \left\{d(g x, g y), d(f x, g x), d(f y, g y), \frac{d(f y, g x)+d(f x, g y)}{2},\right. \\
& \left.\frac{d(f x, g y) d(f y, g x)}{1+d(f x, f y)}, \frac{d(f x, g y) d(f x, g x)}{2[1+d(f x, f y)]}, \frac{d(f y, g x) d(f y, g y)}{2[1+d(f x, f y)]}\right\}, \quad \forall x, y \in X .
\end{aligned}
$$

If $f(X) \subseteq g(X)$ and $g(X)$ is complete, then $f$ and $g$ have a unique common fixed point in $X$.

Proof Firstly we prove that $f$ and $g$ have at most one common fixed point in $X$. Suppose that $f$ and $g$ possess two common fixed points $a, b \in X$ and $a \neq b$. It follows from (2.13), (2.14), and $(\varphi, \psi) \in \Phi \times \Psi$ that

$$
\begin{aligned}
M_{2}(a, b)= & \max \left\{d(g a, g b), d(g a, f a), d(f b, g b), \frac{d(f b, g a)+d(f a, g b)}{2},\right. \\
& \left.\frac{d(f a, g b) d(f b, g a)}{1+d(f a, f b)}, \frac{d(f a, g b) d(f a, g a)}{2[1+d(f a, f b)]}, \frac{d(f b, g a) d(f b, g b)}{2[1+d(f a, f b)]}\right\} \\
= & \max \left\{d(a, b), 0,0, d(a, b), \frac{d^{2}(a, b)}{1+d(a, b)}, 0,0\right\} \\
= & d(a, b)
\end{aligned}
$$

and

$$
\begin{aligned}
\int_{0}^{d(a, b)} \varphi(t) d t & =\int_{0}^{d(f a, f b)} \varphi(t) d t \leq \psi\left(\int_{0}^{M_{2}(a, b)} \varphi(t) d t\right) \\
& =\psi\left(\int_{0}^{d(a, b)} \varphi(t) d t\right) \\
& <\int_{0}^{d(a, b)} \varphi(t) d t,
\end{aligned}
$$

which is absurd. 
Secondly we show that $f$ and $g$ have a common fixed point in $X$. Let $x_{0}$ be an arbitrary point in $X$. Since $f(X) \subseteq g(X)$, it follows that there exists a sequence $\left\{x_{n}\right\}_{n \in \mathbb{N}_{0}}$ in $X$ satisfying $f x_{n}=g x_{n+1}$ for each $n \in \mathbb{N}_{0}$. Put $d_{n}=d\left(f x_{n}, f x_{n+1}\right)$ for all $n \in \mathbb{N}_{0}$.

Assume that $d_{n_{0}}=0$ for some $n \in \mathbb{N}_{0}$. Now we assert that $f x_{n_{0}+1}=f^{2} x_{n_{0}+1}$. Otherwise we infer that in light of (2.2), (2.4), (2.13), (2.14), and $(\varphi, \psi) \in \Phi \times \Psi$

$$
\begin{aligned}
M_{2}\left(f x_{n_{0}+1}, x_{n_{0}+1}\right) & \max \left\{d\left(g f x_{n_{0}+1}, g x_{n_{0}+1}\right), d\left(f^{2} x_{n_{0}+1}, g f x_{n_{0}+1}\right), d\left(f x_{n_{0}+1}, g x_{n_{0}+1}\right),\right. \\
& \frac{d\left(f x_{n_{0}+1}, g f x_{n_{0}+1}\right)+d\left(f^{2} x_{n_{0}+1}, g x_{n_{0}+1}\right)}{2}, \frac{d\left(f^{2} x_{n_{0}+1}, g x_{n_{0}+1}\right) d\left(f x_{n_{0}+1}, g f x_{n_{0}+1}\right)}{1+d\left(f^{2} x_{n_{0}+1}, f x_{n_{0}+1}\right)}, \\
& \left.\frac{d\left(f^{2} x_{n_{0}+1}, g x_{n_{0}+1}\right) d\left(f^{2} x_{n_{0}+1}, g f x_{n_{0}+1}\right)}{2\left[1+d\left(f^{2} x_{n_{0}+1}, f x_{n_{0}+1}\right)\right]}, \frac{d\left(f x_{n_{0}+1}, g f x_{n_{0}+1}\right) d\left(f x_{n_{0}+1}, g x_{n_{0}+1}\right)}{2\left[1+d\left(f^{2} x_{n_{0}+1}, f x_{n_{0}+1}\right)\right]}\right\} \\
= & \max \left\{d\left(f^{2} x_{n_{0}+1}, f x_{n_{0}+1}\right), 0,0, d\left(f^{2} x_{n_{0}+1}, f x_{n_{0}+1}\right),\right. \\
& \left.\frac{d\left(f^{2} x_{n_{0}+1}, f x_{n_{0}+1}\right) d\left(f x_{n_{0}+1}, f^{2} x_{n_{0}+1}\right)}{1+d\left(f^{2} x_{n_{0}+1}, f x_{n_{0}+1}\right)}, 0,0\right\} \\
= & d\left(f^{2} x_{n_{0}+1}, f x_{n_{0}+1}\right)
\end{aligned}
$$

and

$$
\begin{aligned}
\int_{0}^{d\left(f^{2} x_{n_{0}+1}, f x_{n_{0}+1}\right)} \varphi(t) d t & \leq \psi\left(\int_{0}^{M_{2}\left(f x_{n_{0}+1}, x_{n_{0}+1}\right)} \varphi(t) d t\right) \\
& =\psi\left(\int_{0}^{d\left(f^{2} x_{n_{0}+1} f x_{n_{0}+1}\right)} \varphi(t) d t\right) \\
& <\int_{0}^{d\left(f^{2} x_{n_{0}+1} f x_{n_{0}+1}\right)} \varphi(t) d t,
\end{aligned}
$$

which is absurd. Therefore $f x_{n_{0}+1}=f^{2} x_{n_{0}+1}$, which together which (2.3) means that $f x_{n_{0}+1}$ is a common fixed point of $f$ and $g$ in $X$.

Suppose that $d_{n} \neq 0$ for all $n \in \mathbb{N}_{0}$. Using (2.5), (2.13), and (2.14), we deduce that

$$
\begin{aligned}
M_{2}\left(x_{n}, x_{n+1}\right) & \\
= & \max \left\{d\left(g x_{n}, g x_{n+1}\right), d\left(f x_{n}, g x_{n}\right), d\left(f x_{n+1}, g x_{n+1}\right),\right. \\
& \frac{d\left(f x_{n+1}, g x_{n}\right)+d\left(f x_{n}, g x_{n+1}\right)}{2}, \frac{d\left(f x_{n}, g x_{n+1}\right) d\left(f x_{n+1}, g x_{n}\right)}{1+d\left(f x_{n}, f x_{n+1}\right)}, \\
& \left.\frac{d\left(f x_{n}, g x_{n+1}\right) d\left(f x_{n}, g x_{n}\right)}{2\left[1+d\left(f x_{n}, x_{n+1}\right)\right]}, \frac{d\left(f x_{n+1}, g x_{n}\right) d\left(f x_{n+1}, g x_{n+1}\right)}{2\left[1+d\left(f x_{n}, f x_{n+1}\right)\right]}\right\} \\
= & \max \left\{d\left(f x_{n-1}, f x_{n}\right), d\left(f x_{n}, f x_{n-1}\right), d\left(f x_{n+1}, f x_{n}\right),\right. \\
& \frac{d\left(f x_{n+1}, f x_{n-1}\right)+d\left(f x_{n}, f x_{n}\right)}{2}, \frac{d\left(f x_{n}, f x_{n}\right) d\left(f x_{n+1}, f x_{n-1}\right)}{1+d\left(f x_{n}, f x_{n+1}\right)}, \\
& \left.\frac{d\left(f x_{n}, f x_{n}\right) d\left(f x_{n}, f x_{n-1}\right)}{2\left[1+d\left(f x_{n}, f x_{n+1}\right)\right]}, \frac{d\left(f x_{n+1}, f x_{n-1}\right) d\left(f x_{n+1}, f x_{n}\right)}{2\left[1+d\left(f x_{n}, f x_{n+1}\right)\right]}\right\}
\end{aligned}
$$




$$
\begin{aligned}
& =\max \left\{d_{n-1}, d_{n-1}, d_{n}, \frac{d\left(f x_{n+1}, f x_{n-1}\right)}{2}, 0,0, \frac{d\left(f x_{n+1}, f x_{n-1}\right) d_{n}}{2\left(1+d_{n}\right)}\right\} \\
& =\max \left\{d_{n-1}, d_{n}\right\} .
\end{aligned}
$$

If $d_{n}>d_{n-1}$ for some $n \in \mathbb{N}$, making use of (2.13), (2.15), and $(\varphi, \psi) \in \Phi \times \Psi$, we obtain

$$
\begin{aligned}
\int_{0}^{d_{n}} \varphi(t) d t & =\int_{0}^{d\left(f x_{n}, f x_{n+1}\right)} \varphi(t) d t \leq \psi\left(\int_{0}^{M_{2}\left(x_{n}, x_{n+1}\right)} \varphi(t) d t\right) \\
& =\psi\left(\int_{0}^{d_{n}} \varphi(t) d t\right) \\
& <\int_{0}^{d_{n}} \varphi(t) d t
\end{aligned}
$$

which is impossible. Thus $d_{n} \leq d_{n-1}$ for each $n \in \mathbb{N}$. Hence the sequence $\left\{d_{n}\right\}_{n \in \mathbb{N}_{0}}$ is nondecreasing and bounded, which imply that there exists a constant $Q$ with $\lim _{n \rightarrow \infty} d_{n}=Q \geq 0$.

Next we show that $Q=0$. Otherwise $Q>0$. Taking the upper limit in (2.13) and using (2.15), $(\varphi, \psi) \in \Phi \times \Psi$, and Lemma 1.1, we infer that

$$
\begin{aligned}
\int_{0}^{Q} \varphi(t) d t & =\limsup _{n \rightarrow \infty} \int_{0}^{d_{n}} \varphi(t) d t=\limsup _{n \rightarrow \infty} \int_{0}^{d\left(f x_{n}, f x_{n+1}\right)} \varphi(t) d t \\
& \leq \limsup _{n \rightarrow \infty} \psi\left(\int_{0}^{M_{2}\left(x_{n}, x_{n+1}\right)} \varphi(t) d t\right)=\limsup _{n \rightarrow \infty} \psi\left(\int_{0}^{d_{n-1}} \varphi(t) d t\right) \\
& \leq \psi\left(\limsup _{n \rightarrow \infty} \int_{0}^{d_{n-1}} \varphi(t) d t\right)=\psi\left(\int_{0}^{Q} \varphi(t) d t\right) \\
& <\int_{0}^{Q} \varphi(t) d t,
\end{aligned}
$$

which is absurd. Therefore, $Q=0$, that is,

$$
\lim _{n \rightarrow \infty} d_{n}=0, \quad \forall n \in \mathbb{N} .
$$

Now we claim that $\left\{f x_{n}\right\}_{n \in \mathbb{N}_{0}}$ is a Cauchy sequence. Suppose that $\left\{f x_{n}\right\}_{n \in \mathbb{N}_{0}}$ is not a Cauchy sequence. According to (2.10), (2.13), (2.14), (2.16), and $(\varphi, \psi) \in \Phi \times \Psi$ and Lemma 1.1, we have

$$
\begin{aligned}
\lim _{p \rightarrow \infty} & M_{2}\left(x_{m(p)}, x_{n(p)}\right) \\
= & \lim _{p \rightarrow \infty} \max \left\{d\left(g x_{m(p)}, g x_{n(p)}\right), d\left(f x_{m(p)}, g x_{m(p)}\right), d\left(f x_{n(p)}, g x_{n(p)}\right)\right. \\
& \frac{d\left(f x_{n(p)}, g x_{m(p)}\right)+d\left(f x_{m(p)}, g x_{n(p)}\right)}{2}, \frac{d\left(f x_{m(p)}, g x_{n(p)}\right) d\left(f x_{n(p)}, g x_{m(p)}\right)}{1+d\left(f x_{m(p)}, f x_{n(p)}\right)} \\
& \left.\frac{d\left(f x_{m(p)}, g x_{n(p)}\right) d\left(f x_{m(p)}, g x_{m(p)}\right)}{2\left[1+d\left(f x_{m(p)}, f x_{n(p)}\right)\right]}, \frac{d\left(f x_{n(p)}, g x_{m(p)}\right) d\left(f x_{n(p)}, g x_{n(p)}\right)}{2\left[1+d\left(f x_{m(p)}, f x_{n(p)}\right)\right]}\right\} \\
= & \lim _{p \rightarrow \infty} \max \left\{d\left(f x_{m(p)-1}, f x_{n(p)-1}\right), d\left(f x_{m(p)}, f x_{m(p)-1}\right), d\left(f x_{n(p)}, f x_{n(p)-1}\right),\right. \\
& \frac{d\left(f x_{n(p)}, f x_{m(p)-1}\right)+d\left(f x_{m(p)}, f x_{n(p)-1}\right)}{2}, \frac{d\left(f x_{m(p)}, f x_{n(p)-1}\right) d\left(f x_{n(p)}, f x_{m(p)-1}\right)}{1+d\left(f x_{m(p)}, f x_{n(p)}\right)}
\end{aligned}
$$




$$
\begin{aligned}
& \left.\frac{d\left(f x_{m(p)}, f x_{n(p)-1}\right) d\left(f x_{m(p)}, f x_{m(p)-1}\right)}{2\left[1+d\left(f x_{m(p)}, f x_{n(p)}\right)\right]}, \frac{d\left(f x_{n(p)}, f x_{m(p)-1}\right) d\left(f x_{n(p)}, f x_{n(p)-1}\right)}{2\left[1+d\left(f x_{m(p)}, f x_{n(p)}\right)\right]}\right\} \\
= & \max \left\{\varepsilon, 0,0, \varepsilon, \frac{\varepsilon^{2}}{1+\varepsilon}, 0,0\right\} \\
= & \varepsilon
\end{aligned}
$$

and

$$
\begin{aligned}
\int_{0}^{\varepsilon} \varphi(t) d t & \leq \limsup _{p \rightarrow \infty} \int_{0}^{d\left(f x_{m(p)} f x_{n}(p)\right)} \varphi(t) d t \leq \limsup _{p \rightarrow \infty} \psi\left(\int_{0}^{M_{2}\left(x_{m(p)}, x_{n(p)}\right)} \varphi(t) d t\right) \\
& \leq \psi\left(\limsup _{p \rightarrow \infty} \int_{0}^{M_{2}\left(x_{m(p)}, x_{n(p)}\right)} \varphi(t) d t\right) \leq \psi\left(\int_{0}^{\varepsilon} \varphi(t) d t\right) \\
& <\int_{0}^{\varepsilon} \varphi(t) d t,
\end{aligned}
$$

which is a contradiction. Hence $\left\{x_{n}\right\}_{n \in \mathbb{N}}$ is a Cauchy sequence. Since $g(X)$ is complete, there exist $a, z \in X$ such that

$$
\lim _{n \rightarrow \infty} f x_{n}=\lim _{n \rightarrow \infty} g x_{n}=a=g z
$$

Suppose that $f z \neq a$. By means of (2.13), (2.14), (2.18), $(\varphi, \psi) \in \Phi \times \Psi$ and Lemma 1.1, we arrive at

$$
\begin{aligned}
\lim _{n \rightarrow \infty} M_{2}\left(z, x_{n}\right)= & \lim _{n \rightarrow \infty} \max \left\{d\left(g z, g x_{n}\right), d(f z, g z), d\left(f x_{n}, g x_{n}\right),\right. \\
& \frac{d\left(f x_{n}, g z\right)+d\left(f z, g x_{n}\right)}{2}, \frac{d\left(f z, g x_{n}\right) d\left(f x_{n}, g z\right)}{1+d\left(f z, f x_{n}\right)}, \\
& \left.\frac{d\left(f z, g x_{n}\right) d(f z, g z)}{2\left[1+d\left(f z, f x_{n}\right)\right]}, \frac{d\left(f x_{n}, g z\right) d\left(f x_{n}, g x_{n}\right)}{2\left[1+d\left(f z, f x_{n}\right)\right]}\right\} \\
= & \max \left\{d(a, a), d(f z, a), d(a, a), \frac{d(a, a)+d(f z, a)}{2},\right. \\
& \left.\frac{d(f z, a) d(a, a)}{1+d(a, a)}, \frac{d(f z, a) d(f z, a)}{2[1+d(f z, a)]}, \frac{d(a, a) d(a, a)}{2[1+d(f z, a)]}\right\} \\
= & \max \left\{0, d(f z, a), 0, \frac{d(f z, a)}{2}, 0, \frac{d^{2}(f z, a)}{2[1+d(f z, a)]}, 0\right\} \\
= & d(f z, a)
\end{aligned}
$$

and

$$
\begin{aligned}
\int_{0}^{d f f z, a)} \varphi(t) d t & =\limsup _{n \rightarrow \infty} \int_{0}^{d\left(f z, f x_{n}\right)} \varphi(t) d t \leq \limsup _{n \rightarrow \infty} \psi\left(\int_{0}^{M_{2}\left(z, x_{n}\right)} \varphi(t) d t\right) \\
& \leq \psi\left(\limsup _{n \rightarrow \infty} \int_{0}^{M_{2}\left(z, x_{n}\right)} \varphi(t) d t\right)=\psi\left(\int_{0}^{d(f z, a)} \varphi(t) d t\right) \\
& <\int_{0}^{d(f z, a)} \varphi(t) d t,
\end{aligned}
$$


which is a contradiction. Therefore, $a=f z=g z$. Because $f$ and $g$ are weakly compatible, it follows that

$$
f a=f^{2} z=f g z=g f z=g^{2} z=g a .
$$

Suppose that $a \neq f a$. In view of (2.13), (2.14) (2.19), and $(\varphi, \psi) \in \Phi \times \Psi$, we acquire

$$
\begin{aligned}
M_{2}(z, g z)= & \max \left\{d\left(g z, g^{2} z\right), d(f z, g z), d\left(f g z, g^{2} z\right), \frac{d(f g z, g z)+d\left(f z, g^{2} z\right)}{2},\right. \\
& \left.\frac{d\left(f z, g^{2} z\right) d(f g z, g z)}{1+d(f z, f g z)}, \frac{d\left(f z, g^{2} z\right) d(f z, g z)}{2[1+d(f z, f g z)]}, \frac{d(f g z, g z) d\left(f g z, g^{2} z\right)}{2[1+d(f z, f g z)]}\right\} \\
= & \max \left\{d(a, f a), 0,0, \frac{d(f a, a)+d(a, f a)}{2}, \frac{d^{2}(a, f a)}{1+d(a, f a)}, 0,0\right\} \\
= & d(a, f a)
\end{aligned}
$$

and

$$
\begin{aligned}
\int_{0}^{d(a, f a)} \varphi(t) d t & =\int_{0}^{d(f z, f g z)} \varphi(t) d t \leq \psi\left(\int_{0}^{M_{2}(z, g z)} \varphi(t) d t\right) \\
& =\psi\left(\int_{0}^{d(a, f a)} \varphi(t) d t\right) \\
& <\int_{0}^{d(a, f a)} \varphi(t) d t,
\end{aligned}
$$

which is absurd. Hence $a=f a$. It follows from (2.13) that $f$ and $g$ have a common fixed point $a \in X$. This completes the proof.

Similar to the arguments of Theorems 2.1 and 2.2, we conclude the following results and omit their proofs.

Theorem 2.3 Let $(X, d)$ be a metric space and let $f$ and $g$ be weakly compatible selfmappings on $X$ satisfying

$$
\int_{0}^{d(f x, f y)} \varphi(t) d t \leq \psi\left(\int_{0}^{M_{3}(x, y)} \varphi(t) d t\right), \quad \forall x, y \in X
$$

where $(\varphi, \psi) \in \Phi \times \Psi$ and

$$
\begin{aligned}
M_{3}(x, y)= & \max \{d(g x, g y), d(f x, g x), d(f y, g y), \\
& \frac{d(f y, g x)+d(f x, g y)}{2}, \frac{d(f x, g y) d(f y, g x)}{1+d(g x, g y)}, \\
& \left.\min \left\{\frac{d(f x, g x) d(f y, g x)}{1+d(g x, g y)}, \frac{d(f y, g y) d(f x, g y)}{1+d(g x, g y)}\right\}\right\}, \quad \forall x, y \in X .
\end{aligned}
$$

If $f(X) \subseteq g(X)$ and $g(X)$ is complete, then $f$ and $g$ have a unique common fixed point in $X$. 
Theorem 2.4 Let $(X, d)$ be a metric space and let $f$ and $g$ be weakly compatible selfmappings on $X$ satisfying

$$
\int_{0}^{d(f x, f y)} \varphi(t) d t \leq \psi\left(\int_{0}^{M_{4}(x, y)} \varphi(t) d t\right), \quad \forall x, y \in X
$$

where $(\varphi, \psi) \in \Phi \times \Psi$ and

$$
\begin{aligned}
M_{4}(x, y)= & \max \{d(g x, g y), d(f x, g x), d(f y, g y), \\
& \frac{d(f y, g x)+d(f x, g y)}{2}, \frac{d(f x, g y) d(f x, g x)}{1+d(f y, g x)}, \\
& \left.\min \left\{\frac{d(f x, g y) d(f x, g x)}{1+d(f x, f y)}, \frac{d(f y, g x) d(f y, g y)}{1+d(f x, f y)}\right\}\right\}, \quad \forall x, y \in X .
\end{aligned}
$$

If $f(X) \subseteq g(X)$ and $g(X)$ is complete, then $f$ and $g$ have a unique common fixed point in $X$.

Remark 2.5 In case $f=g$ and $\psi(t)=c t$ for each $t \in \mathbb{R}^{+}$, where $c$ is a constant in $(0,1)$, then Theorems 2.1-2.4 reduce to four results which include Theorem 2.1 in [1] and Theorem 2 in [15] as special cases. Example 2.6 below shows that Theorems 2.1-2.4 extend substantially Theorem 2.1 in [1] and Theorem 2 in [15].

Example 2.6 Let $X=\mathbb{R}^{+}$be endowed with the Euclidean metric $d(x, y)=|x-y|$ for all $x, y \in X$. Let $f: X \rightarrow X$ be defined by

$$
f x= \begin{cases}3, & \forall x \in X-\{5\} \\ 4, & x=5\end{cases}
$$

Now we prove that Theorem 2.1 in [1] and Theorem 2 in [15] cannot be used to prove the existence of fixed points of the mapping $f$ in $X$. Suppose that there exist $\varphi \in \Phi$ and $c \in[0,1)$ satisfying the condition of Theorem 2 in [11], that is,

$$
\int_{0}^{d(f x, f y)} \varphi(t) d t \leq c \int_{0}^{m(x, y)} \varphi(t) d t, \quad \forall x, y \in X
$$

where

$$
m(x, y)=\max \left\{d(x, y), d(x, f x), d(y, f y), \frac{1}{2}[d(y, f x)+d(x, f y)]\right\}, \quad \forall x, y \in X .
$$

Taking $\left(x_{0}, y_{0}\right)=(4,5)$ and using $(2.24),(2.25), \varphi \in \Phi$, and $c \in[0,1)$, we get

$$
\begin{aligned}
m\left(x_{0}, y_{0}\right) & =\max \left\{d\left(x_{0}, y_{0}\right), d\left(x_{0}, f x_{0}\right), d\left(y_{0}, f y_{0}\right), \frac{1}{2}\left[d\left(y_{0}, f x_{0}\right)+d\left(x_{0}, f y_{0}\right)\right]\right\} \\
& =\max \left\{d(4,5), d(4,3), d(5,4), \frac{1}{2}[d(5,3)+d(4,4)]\right\} \\
& =1
\end{aligned}
$$


and

$$
\begin{aligned}
\int_{0}^{1} \varphi(t) d t & =\int_{0}^{d\left(f x_{0}, f y_{0}\right)} \varphi(t) d t \leq c \int_{0}^{m\left(x_{0}, y_{0}\right)} \varphi(t) d t=c \int_{0}^{1} \varphi(t) d t \\
& <\int_{0}^{1} \varphi(t) d t
\end{aligned}
$$

which is a contradiction. Note that Theorem 2 in [15] is a generalization of Theorem 2.1 in [1]. Therefore, Theorem 2.1 in [1] is also futile in proving the existence of fixed points for the mapping $f$ in $X$.

Define three mappings $g: X \rightarrow X$ and $\varphi, \psi: \mathbb{R}^{+} \rightarrow \mathbb{R}^{+}$by

$$
\begin{aligned}
& g x= \begin{cases}3, & \forall x \in X \backslash\{5,10\}, \\
10, & x=5, \\
4, & x=10,\end{cases} \\
& \varphi(t)=\frac{\ln (1+t)}{1+t}, \quad \forall t \in \mathbb{R}^{+}
\end{aligned}
$$

and

$$
\psi(t)= \begin{cases}100 \sin t, & \forall t \in[0,1] \\ t^{2}, & \forall t \in(1,+\infty)\end{cases}
$$

It is clear that $(\varphi, \psi) \in \Phi \times \Psi, f$ and $g$ are weakly compatible in $X, f(X)=\{3,4\} \subseteq$ $\{3,4,10\}=g(X)$ and $g(X)$ is complete. Let $x, y \in X$ with $x<y$. In order to verify (2.1), (2.3), (2.20), and (2.22) hold, we have to consider the following four possible cases:

Case 1. $x, y \in X \backslash\{5\}$. It follows that

$$
\int_{0}^{d(f x, f y)} \varphi(t) d t=\int_{0}^{d(3,3)} \varphi(t) d t=0 \leq \psi\left(\int_{0}^{M_{i}(x, y)} \varphi(t) d t\right), \quad \forall i \in\{1,2,3,4\}
$$

Case 2. $x=5$ and $y=10$. Observe that $\psi$ is strictly increasing in $(1,+\infty)$ and

$$
M_{i}(x, y) \geq d(g x, g y)=d(10,4)=6, \quad \forall i \in\{1,2,3,4\}
$$

It is easy to verify that

$$
\begin{aligned}
\int_{0}^{d(f x, f y)} \varphi(t) d t & =\int_{0}^{d(4,3)} \varphi(t) d t=\int_{0}^{1} \varphi(t) d t=\frac{1}{2} \ln ^{2} 2<\frac{1}{4}<1 \\
& <\frac{1}{4} \ln ^{4} 7=\psi\left(\frac{1}{2} \ln ^{2} 7\right)=\psi\left(\int_{0}^{6} \varphi(t) d t\right) \\
& \leq \psi\left(\int_{0}^{M_{i}(x, y)} \varphi(t) d t\right), \quad \forall i \in\{1,2,3,4\} .
\end{aligned}
$$

Case 3. $x=5$ and $y \in(5,10) \cup(10,+\infty)$. Note that $\psi$ is strictly increasing in $(1,+\infty)$ and

$$
M_{i}(x, y) \geq d(g x, g y)=d(10,3)=7, \quad \forall i \in\{1,2,3,4\} .
$$


It is clear that

$$
\begin{aligned}
\int_{0}^{d(f x, f y)} \varphi(t) d t & =\int_{0}^{d(4,3)} \varphi(t) d t=\int_{0}^{1} \varphi(t) d t=\frac{1}{2} \ln ^{2} 2<\frac{1}{4}<1 \\
& <\frac{1}{4} \ln ^{4} 8=\psi\left(\frac{1}{2} \ln ^{2} 8\right)=\psi\left(\int_{0}^{7} \varphi(t) d t\right) \\
& \leq \psi\left(\int_{0}^{M_{i}(x, y)} \varphi(t) d t\right), \quad \forall i \in\{1,2,3,4\} .
\end{aligned}
$$

Case 4. $x<5$ and $y=5$. Note that $\psi$ is strictly increasing in $(1,+\infty)$ and

$$
M_{i}(x, y) \geq d(g x, g y)=d(3,10)=7, \quad \forall i \in\{1,2,3,4\} .
$$

Clearly we have

$$
\begin{aligned}
\int_{0}^{d(f x, f y)} \varphi(t) d t & =\int_{0}^{d(3,4)} \varphi(t) d t=\int_{0}^{1} \varphi(t) d t=\frac{1}{2} \ln ^{2} 2<\frac{1}{4}<1 \\
& <\frac{1}{4} \ln ^{4} 8=\psi\left(\frac{1}{2} \ln ^{2} 8\right)=\psi\left(\int_{0}^{7} \varphi(t) d t\right) \\
& \leq \psi\left(\int_{0}^{M_{i}(x, y)} \varphi(t) d t\right), \quad \forall i \in\{1,2,3,4\} .
\end{aligned}
$$

That is, (2.1), (2.3), (2.20), and (2.22) hold. Hence each of Theorems 2.1-2.4 guarantees that $f$ and $g$ possess a unique common fixed point in $X$.

Finally, we use Theorem 2.1 to discuss solvability of the following system of functional equations arising in dynamic programming:

$$
\begin{array}{ll}
f(x)=\operatorname{opt}_{y \in D}\{u(x, y)+H(x, y, f(a(x, y)))\}, & \forall x \in S, \\
g(x)=\operatorname{opt}_{y \in D}\{v(x, y)+G(x, y, g(b(x, y)))\}, & \forall x \in S,
\end{array}
$$

where opt stands for sup or inf, $Z$ and $Y$ are Banach spaces, $S \subseteq Z$ is the state space, $D \subseteq Y$ is the decision space, $x$ and $y$ signify the state and decision vectors, respectively, $a$ and $b$ represent the transformations of the process, $f(x)$ and $g(x)$ denote the optimal return functions with the initial state $x, B(S)$ denotes the Banach space of all bounded real-valued functions on $S$ with norm

$$
\|w\|=\sup \{|w(x)|: x \in S\} \quad \text { for any } w \in B(S)
$$

Example 2.7 Let $u, v: S \times D \rightarrow \mathbb{R}, a, b: S \times D \rightarrow S, H, G: S \times D \times \mathbb{R} \rightarrow \mathbb{R}$ and $(\varphi, \psi) \in$ $\Phi \times \Psi$ satisfy the following conditions:

$u, v, H$ and $G$ are bounded;

$f g h=g f h \quad$ for each $h \in B(S)$ with $f h=g h$;

$f(B(S)) \subseteq g(B(S))$ and $g(B(S))$ are complete 
and

$$
\begin{aligned}
& \int_{0}^{|H(x, y, h(a(x, y)))-H(x, y, w(a(x, y)))|} \varphi(t) d t \leq \psi\left(\int_{0}^{M_{1}^{*}(h, w)} \varphi(t) d t\right), \\
& \forall(x, y, h, w) \in S \times D \times B(S) \times B(S),
\end{aligned}
$$

where the mappings $f$ and $g$ are defined by

$$
\begin{array}{ll}
f h(x)=\operatorname{opt}_{y \in D}\{u(x, y)+H(x, y, h(a(x, y)))\}, & \forall(x, h) \in S \times B(S), \\
g h(x)=\operatorname{opt}_{y \in D}\{v(x, y)+G(x, y, h(b(x, y)))\}, & \forall(x, h) \in S \times B(S)
\end{array}
$$

and

$$
\begin{aligned}
M_{1}^{*}(h, w)= & \max \{\|g h-g w\|,\|f h-g h\|,\|f w-g w\|, \\
& \frac{\|f h-g w\|+\|f w-g h\|}{2}, \frac{\|f h-g w\|\|f w-g h\|}{1+\|g h-g w\|}, \\
& \left.\frac{\|f h-g h\|\|f w-g h\|}{2(1+\|g h-g w\|)}, \frac{\|f w-g w\|\|f h-g w\|}{2(1+\|g h-g w\|)}\right\}, \quad \forall h, w \in B(S) .
\end{aligned}
$$

Then the system of functional equations (2.26) has a unique common solution $w \in B(S)$.

Proof It follows from (2.27) that there exists $M>0$ satisfying

$$
\sup \{|u(x, y)|,|v(x, y)|,|H(x, y, t)|,|G(x, y, t)|:(x, y, t) \in S \times D \times \mathbb{R}\} \leq M
$$

It is easy to see that $f$ and $g$ are self-mappings in $B(S)$ by (2.31), (2.33), and Lemma 1.2. It is clear that Theorem 12.34 in [24] and $\varphi \in \Phi$ yield, for each $\varepsilon>0$ : there exists $\delta \in(0, M)$ satisfying

$$
\int_{C} \varphi(t) d t<\varepsilon, \quad \forall C \subseteq[0,3 M] \text { with } m(C) \leq \delta,
$$

where $m(C)$ denotes the Lebesgue measure of $C$.

Let $(x, h, w) \in S \times B(S) \times B(S)$. Suppose that opt ${ }_{y \in D}=\inf _{y \in D}$. Clearly (2.31) implies that there exist $y, z \in D$ satisfying

$$
\begin{aligned}
& f h(x)>u(x, y)+H(x, y, h(a(x, y)))-\delta \\
& f w(x)>u(x, z)+H(x, z, w(a(x, z)))-\delta \\
& f h(x) \leq u(x, z)+H(x, z, h(a(x, z))) \\
& f w(x) \leq u(x, y)+H(x, y, w(a(x, y)))
\end{aligned}
$$

which means that

$$
\begin{aligned}
f h(x)-f w(x)> & H(x, y, h(a(x, y)))-H(x, y, w(a(x, y)))-\delta \\
\geq & -\max \{|H(x, y, h(a(x, y)))-H(x, y, w(a(x, y)))|, \\
& |H(x, z, h(a(x, z)))-H(x, z, w(a(x, z)))|\}-\delta
\end{aligned}
$$


and

$$
\begin{aligned}
f h(x)-f w(x)< & H(x, z, h(a(x, z)))-H(x, z, w(a(x, z)))+\delta \\
\leq & \max \{|H(x, y, h(a(x, y)))-H(x, y, w(a(x, y)))|, \\
& |H(x, z, h(a(x, z)))-H(x, z, w(a(x, z)))|\}+\delta,
\end{aligned}
$$

which yield

$$
\begin{aligned}
|f h(x)-f w(x)|< & \max \{|H(x, y, h(a(x, y)))-H(x, y, w(a(x, y)))|, \\
& |H(x, z, h(a(x, z)))-H(x, z, w(a(x, z)))|\}+\delta \\
= & \max \{|H(x, y, h(a(x, y)))-H(x, y, w(a(x, y)))|+\delta, \\
& |H(x, z, h(a(x, z)))-H(x, z, w(a(x, z)))|+\delta\} .
\end{aligned}
$$

Similarly we infer that (2.35) holds also for opt $\mathrm{y}_{y \in D}=\sup _{y \in D}$. Combining (2.31), (2.34), and (2.35), we arrive at

$$
\begin{aligned}
\int_{0}^{|f h(x)-f w(x)|} \varphi(t) d t \leq & \max \left\{\int_{0}^{|H(x, y, h(a(x, y)))-H(x, y, w(a(x, y)))|+\delta} \varphi(t) d t\right. \\
& \left.\int_{0}^{|H(x, z, h(a(x, z)))-H(x, z, w(a(x, z)))|+\delta} \varphi(t) d t\right\} \\
= & \max \left\{\int_{0}^{|H(x, y, h(a(x, y)))-H(x, y, w(a(x, y)))|} \varphi(t) d t\right. \\
& +\int_{|H(x, y, h(a(x, y)))-H(x, y, w(a(x, y)))|}^{|H(x, y, h(a(x, y)))-H(x, y, w(a(x, y)))|+\delta} \varphi(t) d t, \\
& \int_{0}^{\mid H(x, z, h(a(x, z)))-H(x, z, w(a(x, z)) \mid} \varphi(t) d t \\
& \left.+\int_{|H(x, z, h(a(x, z)))-H(x, z, w(a(x, z)))|}^{\mid H(x, z, h(a(x, z)))-H(x, z, w(a(x, z)) \mid+\delta} \varphi(t) d t\right\} \\
\leq & \max \left\{\int_{0}^{|H(x, y, h(a(x, y)))-H(x, y, w(a(x, y)))|} \varphi(t) d t,\right. \\
& \left.\int_{0}^{|H(x, z, h(a(x, z)))-H(x, z, w(a(x, z)))|} \varphi(t) d t\right\} \\
& +\max \left\{\int_{|H(x, y, h(a(x, y)))-H(x, y, w(a(x, y)))|}^{\mid H(x, y, h(a(x, y))-H(x, y, w(a(x, y))) \mid+\delta} \varphi(t) d t,\right. \\
& \left.\int_{|H(x, z, h(a(x, z)))-H(x, z, w(a(x, z)))|}^{\mid H(x, z, h(a(x, z)))-H(x, z, w(a(x, z)) \mid+\delta} \varphi(t) d t\right\} \\
\leq & \psi\left(\int_{0}^{M} \varphi(t(h, w) d t)+\varepsilon, \quad \forall(x, h, w) \in S \times B(S) \times B(S),\right. \\
&
\end{aligned}
$$

which means that

$$
\int_{0}^{\|f h-f w\|} \varphi(t) d t \leq \psi\left(\int_{0}^{M_{1}^{*}(h, w)} \varphi(t) d t\right)+\varepsilon, \quad \forall h, w \in B(S),
$$


letting $\varepsilon \rightarrow 0^{+}$in the above inequality, we deduce that

$$
\int_{0}^{\|f h-f w\|} \varphi(t) d t \leq \psi\left(\int_{0}^{M_{1}^{*}(h, w)} \varphi(t) d t\right), \quad \forall h, w \in B(S)
$$

Thus Theorem 2.1 ensures that the mappings $f$ and $g$ have a unique common fixed point $w \in B(S)$, which is a unique common solution of the system of functional equations (2.26). This completes the proof.

Remark 2.8 The conclusion of Example 2.7 generalizes and improves Theorems 5.1-5.3 in [12], Theorem 1 in [21], Theorem 3.1 in [22], and Theorem 3.2 in [23].

\section{Competing interests}

The authors declare that they have no competing interests.

\section{Authors' contributions}

All authors read and approved the final manuscript.

\section{Author details}

'Department of Mathematics, Liaoning Normal University, Dalian, Liaoning 116029, People's Republic of China. ${ }^{2}$ Department of Mathematics and the RINS, Gyeongsang National University, Jinju, 660-701, Korea. ${ }^{3}$ Department of Applied Mathematics, Changwon National University, Changwon, 641-773, Korea.

\section{Acknowledgements}

The authors thank the referees for useful comments and suggestions. This research was supported by the Science Research Foundation of Educational Department of Liaoning Province (L2012380) and Basic Science Research Program through the National Research Foundation of Korea (NRF) funded by the Ministry of Science, ICT \& Future Planning (2013R1A1A2057665).

\section{Received: 18 March 2014 Accepted: 18 September 2014 Published: 16 October 2014}

\section{References}

1. Branciari, A: A fixed point theorem for mappings satisfying a general contractive condition of integral type. Int. J. Math. Math. Sci. 29, 531-536 (2002). doi:10.1155/S0161171202007524

2. Aliouche, A: A common fixed point theorem for weakly compatible mappings in symmetric spaces satisfying a contractive condition of integral type. J. Math. Anal. Appl. 322, 796-802 (2006). doi:10.1016/j.jmaa.2005.09.068

3. Altun, I, Türkoǧlu, D: Some fixed point theorems for weakly compatible mappings satisfying an implicit relation. Taiwan. J. Math. 13, 1291-1304 (2009)

4. Altun, I, Türkoǧlu, D: Some fixed point theorems for mappings satisfying contractive condition of integral type on d-complete topological spaces. Fasc. Math. 42, 5-15 (2009)

5. Altun, I, Türkoǧlu, D, Rhoades, BE: Fixed points of weakly compatible maps satisfying a general contractive of integral type. Fixed Point Theory Appl. 2007, Article ID 17301 (2007). doi:10.1155/2007/17301

6. Beygmohammadi, M, Razani, A: Two fixed-point theorems for mappings satisfying a general contractive condition of integral type in the modular space. Int. J. Math. Math. Sci. 2010, Article ID 317107 (2010). doi:10.1155/2010/317107

7. Djoudi, A, Aliouche, A: Common fixed point theorems of Greguš type for weakly compatible mappings satisfying contractive conditions of integral type. J. Math. Anal. Appl. 329, 31-45 (2007). doi:10.1016/j.jmaa.2006.06.037

8. Djoudi, A, Merghadi, F: Common fixed point theorems for maps under a contractive condition of integral type. J. Math. Anal. Appl. 341, 953-960 (2008). doi:10.1016/j.jmaa.2007.10.064

9. Hussain, N, Salimi, P: Implicit contractive mappings in modular metric and fuzzy metric spaces. Sci. World J. 2014, Article ID 981578 (2014). doi:10.1155/2014/981578

10. Jachymski, J: Remarks on contractive conditions of integral type. Nonlinear Anal. 71, 1073-1081 (2009). doi:10.1016/j.na.2008.11.046

11. Khojasteh, F, Goodarzi, Z, Razani, A: Some fixed point theorems of integral type contraction in cone metric spaces. Fixed Point Theory Appl. 2010, Article ID 189684 (2010). doi:10.1155/2010/189684

12. Liu, Z, Li, X, Kang, SM, Cho, SY: Fixed point theorems for mappings satisfying contractive conditions of integral type and applications. Fixed Point Theory Appl. 2011, 64 (2011). doi:10.1186/1687-1812-2011-64

13. Mongkolkeha, C, Kumam, P: Fixed point and common fixed point theorems for generalized weak contraction mappings of integral type in modular spaces. Int. J. Math. Math. Sci. 2011, Article ID 705943 (2011). doi:10.1155/2011/705943

14. Murthy, PP, Kumar, S, Tas, K: Common fixed points of self maps satisfying an integral type contractive condition in fuzzy metric spaces. Math. Commun. 15, 521-537 (2010)

15. Rhoades, BE: Two fixed-point theorems for mappings satisfying a general contractive condition of integral type. Int J. Math. Math. Sci. 63, 4007-4013 (2003). doi:10.1155/S0161171203208024

16. De la Sen, M: Fixed point and best proximity theorems under two classes of integral-type contractive conditions in uniform metric spaces. Fixed Point Theory Appl. 2010, Article ID 510974 (2010). doi:10.1155/2010/510974 
17. Sintunavarat, W, Kumam, P: Gregus-type common fixed point theorems for tangential multi-valued mappings of integral type in metric spaces. Int. J. Math. Math. Sci. 2011, Article ID 923458 (2011). doi:10.1155/2011/923458

18. Sintunavarat, W, Kumam, P: Gregus-type fixed points for a tangential multi-valued mappings satisfying contractive conditions of integral type. J. Inequal. Appl. 2011, 3 (2011). doi:10.1186/1029-242X-2011-3

19. Suzuki, T: Meir-Keeler contractions of integral type are still Meir-Keeler contractions. Int. J. Math. Math. Sci. 2007, Article ID 39281 (2007). doi:10.1155/2007/39281

20. Vijayaraju, P, Rhoades, BE, Mohanraj, R: A fixed point theorem for a pair of maps satisfying a general contractive condition of integral type. Int. J. Math. Math. Sci. 15, 2359-2364 (2005). doi:10.1155/IJMMS.2005.2359

21. Bhakta, PC, Mitra, S: Some existence theorems for functional equations arising in dynamic programming. J. Math. Anal. Appl. 98, 348-362 (1984). doi:10.1016/0022-247X(84)90254-3

22. Liu, Z, Kang, SM: Existence and uniqueness of solutions for two classes of functional equations arising in dynamic programming. Acta Math. Appl. Sinica (Engl. Ser.) 23, 195-208 (2007). doi:10.1007/s10255-007-0363-6

23. Liu, Z, Xu, YG, Ume, JS, Kang, SM: Solutions to two functional equations arising in dynamic programming. J. Comput. Appl. Math. 192, 251-269 (2006). doi:10.1016/j.cam.2005.04.033

24. Hewitt, E, Stromberg, K: Real and Abstract Analysis. Springer, New York (1978)

doi:10.1186/1029-242X-2014-394

Cite this article as: Liu et al.: Common fixed points for a pair of mappings satisfying contractive conditions of integral type. Journal of Inequalities and Applications 2014 2014:394.

\section{Submit your manuscript to a SpringerOpen ${ }^{\circ}$ journal and benefit from:}

- Convenient online submission

- Rigorous peer review

- Immediate publication on acceptance

Open access: articles freely available online

- High visibility within the field

- Retaining the copyright to your article 